\title{
BMJ Open The renal parenchyma-evaluation of a novel ultrasound measurement to assess fetal renal development: protocol for an observational longitudinal study
}

\author{
Sonja Brennan, ${ }^{1,2}$ Michal Schneider, ${ }^{3}$ David Watson, ${ }^{2,4}$ Yogavijayan Kandasamy, ${ }^{2,5,6}$ \\ Donna Rudd ${ }^{2}$
}

To cite: Brennan S,

Schneider M, Watson D, et al.

The renal parenchyma-

evaluation of a novel ultrasound measurement to assess

fetal renal development: protocol for an observational longitudinal study. BMJ Open 2017;7:e019369. doi:10.1136/ bmjopen-2017-019369

- Prepublication history for this paper is available online. To view these files, please visit the journal online (http://dx.doi. org/10.1136/bmjopen-2017019369).

Received 5 September 2017 Revised 4 November 2017 Accepted 24 November 2017

CrossMark

For numbered affiliations see end of article.

Correspondence to

Sonja Brennan;

sonja.brennan@my.jcu.edu.au

\section{ABSTRACT}

Introduction Disorders of fetal growth, such as intrauterine growth restriction (IUGR) and large for gestational age (LGA), have been found to have a profound effect on the development of the fetal kidney. Abnormal kidney development is associated with hypertension and chronic kidney disease later in life. This study will use a novel ultrasound measurement to assess the renal parenchymal growth and kidney arterial blood flow in the fetus to evaluate the development of the fetal kidneys and provide an indirect estimate of nephron number. Measurements in normally grown, IUGR and LGA fetuses will be compared to determine if changes in renal parenchymal growth can be detected in utero.

Methods and analysis This longitudinal, prospective, observational study will be conducted over 12 months in the Ultrasound Department of the Townsville Hospital, Australia. The study will compare fetal renal parenchymal thickness (RPT) and renal artery Doppler flow between IUGR fetuses and appropriately grown fetuses, and LGA fetuses and appropriately grown fetuses between 16 and 40 weeks. The fetal RPT to renal volume ratio will also be compared, and correlations between RPT, renal parenchymal echogenicity, fetal Doppler indices and amniotic fluid levels will be analysed.

Ethics and dissemination This study was approved by the Townsville Health District Human Research Ethics Committee. The study results will form part of a thesis and will be published in peer-reviewed journals and disseminated at international conferences.

\section{INTRODUCTION}

Chronic kidney disease is an increasing contributor to the global burden of disease, with hypertension now the leading risk factor. ${ }^{1}$ Recognition of the risk factors and implementation of preventive strategies is crucial to reducing hypertension and kidney disease. Abnormalities in fetal growth, such as intrauterine growth restriction (IUGR), have a profound effect on the kidney development. ${ }^{23}$ The association between an adverse intrauterine environment and chronic kidney disease later in life is now compelling. ${ }^{4-6}$
Strengths and limitations of this study

- This will be the first study to use a novel ultrasound measurement of the fetal renal parenchyma and measurements of renal blood flow to assess fetal kidney growth.

- Fetal kidney growth will be assessed not only in normally grown but also in growth-restricted and large-for-gestational-age fetuses.

- This is a prospective, longitudinal, rather than crosssectional, ultrasound study and should enhance our understanding of how fetal kidneys grow.

- This study is the first of a series of studies investigating kidney growth, and although renal function and renal parenchymal thickness of the infants are not included in this study, follow-up of the infants will be included in future studies.

- Due to fetal position and/or maternal habitus, not all kidney measurements may be obtainable at every scan.

During early fetal life, there is transient development and regression of the primary (pronephros) and secondary (mesonephros) fetal kidneys between day 23 and day 112 of embryonic life. ${ }^{7}$ The permanent functional tertiary fetal kidney is the metanephros which begins developing on day $30 .{ }^{7}$ Nephrogenesis involves the formation of the functional units of the kidney called nephrons and continues up to 36 weeks' gestational age. ${ }^{89}$ It is essential that appropriate nephrogenesis is achieved in utero as the number and quality of nephrons directly influences lifetime kidney function. ${ }^{10}$

IUGR can result in a significant reduction in nephron number; however, large for gestational age (LGA), particularly related to maternal hyperglycaemia, is also associated with abnormal fetal kidney development and an increased risk of hypertension and chronic kidney disease. ${ }^{11}$ A better understanding of the relationship between abnormal fetal growth and nephrogenesis is needed. 


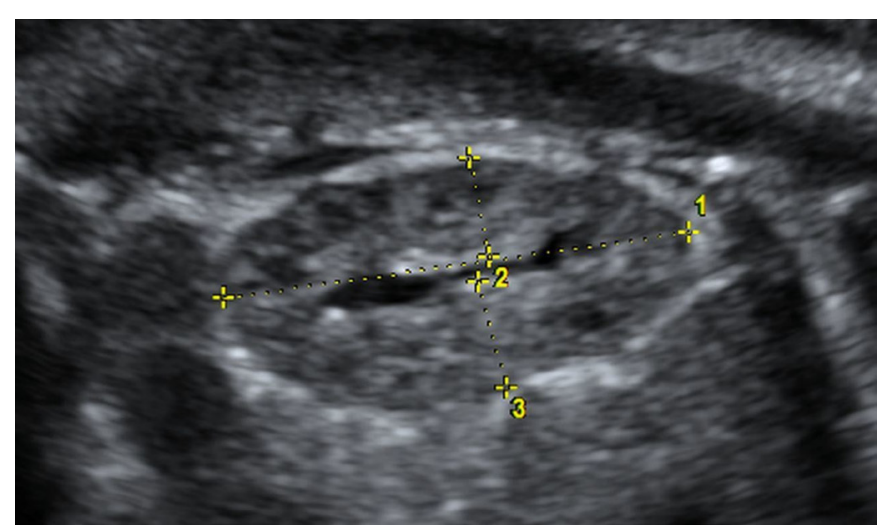

Figure 1 Measurement of kidney length (1) and the anterior (2) and posterior (3) fetal renal parenchymal thickness from the inner aspect of the renal capsule to the sinus-pyramidal apex interface.

Presently, the only accurate method of calculating human nephron number is during an autopsy. ${ }^{12}$ A non-invasive measure of nephron endowment is needed.

Ultrasound is the primary imaging modality for evaluating fetal kidneys. We conducted a systematic review on the evaluation of fetal kidney growth using ultrasound which revealed that there are few good-quality, longitudinal studies. ${ }^{13}$ The most commonly reported ultrasound measurement was renal length; however, renal length alone was not found to be very sensitive to evaluate disruptions in fetal kidney growth in the presence of fetal growth restriction. ${ }^{13}$ Few studies analysed the effects of IUGR on fetal kidney growth, and no studies have, to date, analysed if LGA has an effect on fetal kidney growth. Results from two-dimensional (2D) and three-dimensional (3D) renal volume (RV) calculations were disappointing. Volumes calculated from 2D measurements underestimate RVs by as much as $24 \% .{ }^{14}$ Substantial variations were reported for 'normal' 3D kidney volumes, and good reliability and reproducibility has not yet been demonstrated. Currently, there is no easily repeatable, sensitive method of measuring changes in fetal kidney growth. ${ }^{13}$

Measuring the renal parenchyma with ultrasound is a novel method to assess fetal kidney development and predict future renal function. Measuring just the renal parenchyma will measure only the important functional part of the kidney which contains the nephrons (figure 1). One small cross-sectional study measured fetal renal parenchyma in normally grown fetuses ${ }^{15}$; however, no studies have evaluated the fetal renal parenchyma in abnormally grown fetuses. Preliminary data from term neonates ${ }^{16}$ and children ${ }^{17}$ indicate that the parenchymal thickness may be a more reliable investigative method to define normal and abnormal kidney development. Methods such as measuring the parenchymal thickness, RV to parenchymal thickness ratio, renal artery Dopplers and echogenicity of the renal parenchyma are potential non-invasive methods to evaluate nephron endowment and future renal function.
The aim of this study is to use ultrasound to assess the fetal renal parenchymal growth in a pregnant population demonstrating either normal or abnormal growth and determine if abnormal fetal growth influences fetal renal parenchymal thickness (RPT). Non-invasive ultrasound techniques are used. The results could help identify factors that adversely affect kidney development so that they could be modified by public health interventions and education programmes. This may promote improved fetal nephron number and quality at birth and reduce susceptibility to chronic disease in later life.

\section{METHODS AND ANALYSIS \\ Objectives}

Primary objectives

- Determine normal RPT of fetuses from 16 to 40 weeks' gestation from a group of normally grown fetuses.

- Determine the effects of IUGR and LGA on RPT in a group of abnormally grown fetuses.

Secondary objectives

- Assess the relationship between RPT and renal artery, umbilical artery and middle cerebral artery Doppler indices, and amniotic fluid levels.

- Assess the relationship between renal parenchymal echogenicity, renal artery Doppler flow and IUGR or LGA.

\section{Study design and setting}

This is a prospective, longitudinal, observational study being conducted over 12 months, commencing in May 2017, in the Ultrasound Department of the Townsville Hospital, Australia.

\section{Participants}

Patients who are referred for a diagnostic second trimester ultrasound scan will be recruited for this study. Pregnant women of 18 years or older, with an accurately dated singleton pregnancy of 16 weeks' gestation or more, will be included. Pregnant women with uncertain dates, multiple pregnancy or any known major congenital fetal abnormality or chromosomal fetal abnormality will be excluded.

\section{Recruitment and consent of participants}

Pregnant patients 18 years of age or older, who present to the Medical Imaging Department at the Townsville Hospital for an obstetric ultrasound, will be invited to participate. In addition, mixed risk patients may also be informed about the study by their treating obstetrician, midwife or sonographer. Detailed written information will be given to the patient and written consent obtained.

\section{Study process}

Figure 2 outlines the flow schedule of the participants. The first ultrasound scan will be performed from 16 weeks and then follow-up ultrasounds will be performed at least every 4 weeks from the first ultrasound scan. Some women, particularly with high-risk pregnancies, will 


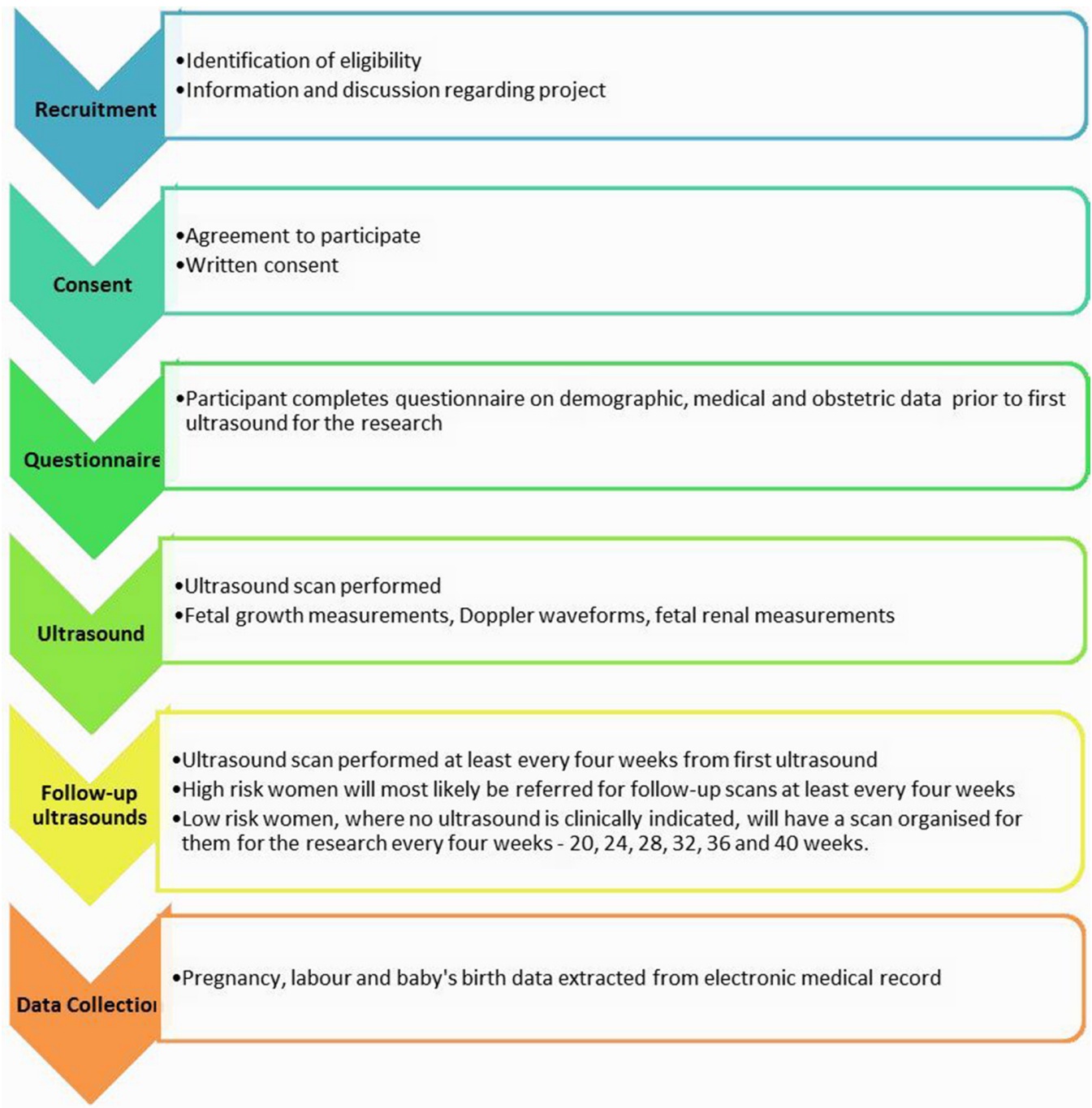

Figure 2 Flow chart of study participants.

require more than one clinically indicated ultrasound. For example, women with a growth-restricted fetus may need to be monitored by ultrasound monthly or more frequently. However, the control group of healthy women, with appropriately grown fetuses, may only require one to two clinically indicated scans between 16 and 40 weeks' gestation. To obtain good longitudinal data, particularly for the control group, the women will be asked to attend for additional research scans every 4 weeks until delivery.

\section{Ultrasound examinations}

Australian Accredited Medical Sonographers with at least 2years' postultrasound qualification experience will perform all ultrasound examinations. A high-level ultrasound machine with pulse wave and colour Doppler flow will be used, and the highest frequency transducer possible, matching the mother's body habitus, will be selected. When a woman attends for a clinically indicated scan, this will be the priority, and then the additional fetal renal measurements required for the research study will be performed thereafter.

Where possible, the fetal kidneys should be measured with the fetal spine up (anterior) or as close as possible to this position. The image is magnified so that the kidney occupies most of the image and both kidneys can be identified. A midsagittal scan of both kidneys along their longest length will be recorded and the longest length (L) of both kidneys measured. The parenchymal thickness will be measured in two directions: from the posterior aspect of the kidney to the pelvis (posterior parenchyma) and from the anterior border of the kidney to the pelvis (anterior parenchyma) (figure 1). A transverse section of the fetal abdomen at the level of each renal pelvis will be imaged. The maximum anteroposterior diameter $(\mathrm{H})$ and transverse diameter (W) will be measured for both kidneys.

Bilateral fetal renal artery Dopplers will be performed in the coronal view of the kidneys. Colour flow should be used to identify the renal artery entering the kidney. A low wall filter of between 30 and $60 \mathrm{~Hz}$ will be used, and a sample gate of size $2-3 \mathrm{~mm}$ will be placed in the mid trunk of the renal artery. Using an angle as close to $0^{\circ}$ as possible, a pulse wave signal will be obtained. The average of three consecutive waveforms will be used to calculate the resistivity index (RI) and pulsatility index (PI). 
Table 1 After baby's birth, perinatal data to be collected from the mother and baby's electronic medical record

Birth data to be collected

\begin{tabular}{|c|c|c|}
\hline Onset of labour & $\begin{array}{l}\text { Gestational age } \\
\text { at birth }\end{array}$ & Antenatal steroids \\
\hline $\begin{array}{l}\text { Mode of } \\
\text { delivery }\end{array}$ & Birth weight & $\begin{array}{l}\text { Other antenatal } \\
\text { medications }\end{array}$ \\
\hline \multirow[t]{5}{*}{$\begin{array}{l}\text { Placental } \\
\text { histopathology }\end{array}$} & Gender & $\begin{array}{l}\text { Maternal medical } \\
\text { history: }\end{array}$ \\
\hline & $\begin{array}{l}\text { Apgar scores at } 1 \\
\text { and } 5 \mathrm{~min}\end{array}$ & Diabetes \\
\hline & $\begin{array}{l}\text { Umbilical artery } \\
\text { cord potential } \\
\text { hydrogen }(\mathrm{pH})\end{array}$ & - Renal disease \\
\hline & Base excess & - Hypertension \\
\hline & Lactate & $\begin{array}{l}\text { Demographic, medical } \\
\text { and obstetric history } \\
\text { from participant } \\
\text { questionnaire }\end{array}$ \\
\hline
\end{tabular}

The following routinely performed obstetric measurements will also be recorded for the study:

- Single deepest pool amniotic fluid measurement;

- Umbilical artery Doppler;

- Middle cerebral artery Doppler (where clinically indicated or 30 weeks' gestation and over);

- Ductus venous, where clinically indicated;

- Biometries-head circumference (HC), biparietal diameter (BPD), abdominal circumference (AC), femur length (FL).

\section{Birth data}

\section{Outcome measures}

Perinatal data will be collected from the mother and baby's electronic medical record (table 1).

Primary outcome measure

- RPT: anterior and posterior thickness in longitudinal plane.

Secondary outcome measures

- RV: calculated using the formula $\mathrm{RV}=$ length $\times$ width $\times$ height $\times 0.523$;

- Fetal growth biometries: HC, BPD, AC and FL;

- Amniotic fluid: single deepest pool;

- Umbilical artery Doppler flow: RI and PI calculated from the average of at least three consecutive waveforms;

- Middle cerebral artery Doppler flow: RI and PI calculated from the average of at least three consecutive waveforms;

- Renal parenchymal echogenicity: subjectively assessed by the sonographer as either normal echogenicity, more hyperechoic than normal or more hypoechoic than normal;

- Renal artery Doppler flow: RI and PI calculated from the average of at least three consecutive waveforms.

\section{Sample size}

Optimal sample size has been calculated based on a statistical power of $80 \%$ and a significance level of 0.05 (two-tailed). Data from a previously published study ${ }^{16}$ have demonstrated that the RPT was $9.4 \mathrm{~mm}( \pm 1.1 \mathrm{~mm})$ for normal birth weight neonates and $8.3 \mathrm{~mm}( \pm 1.0 \mathrm{~mm})$ for low birth weight neonates at term. Therefore, it is estimated that a sample size of 45 will be needed (15 intrauterine growth-restricted fetuses, 15 LGA fetuses and 15 appropriate for gestational age (AGA)). Allowing for the possibility of loss to follow-up, 20 participants will be recruited for each group resulting in a total of 60 participants, each having an ultrasound scan every 4 weeks.

\section{Data analysis}

After the ultrasound examination is complete, and the baby is born, all data will be collated and divided into three groups: AGA, IUGR and LGA. Comparisons of RPT and renal artery Dopplers between IUGR and AGA fetuses, and LGA and AGA fetuses between 16 and 40 weeks will be analysed. RV will be compared with RPT in each fetal group to obtain an RPT to RV ratio. Correlation between RPT, renal parenchymal echogenicity, fetal Doppler indices and amniotic fluid levels will be carried out, and intraobserver and interobserver variability will be assessed.

Statistical analysis will be performed using IBM SPSS Statistics, V.24.0. The normality of the variables will be determined by the Kolmogorov-Smirnov test. Renal measurements will be expressed as means \pm SDs for continuous, normally distributed data and as a median (IQR) for continuous, non-normally distributed data. Paired/unpaired t-tests will be used to compare means of normally distributed data and Mann-Whitney or Kruskal-Wallis tests for non-normally distributed data. A value of $P<0.05$ will be considered statistically significant. Univariate and multivariate analysis will be carried out to determine the association between RPT and other variables. Intraobserver variability will be determined by calculating the differences between the two measurements made by the same sonographer. Interobserver variability will be assessed by calculating the differences between two measurements carried out on the same patient by different sonographers.

\section{Data management}

Data collection commenced in May 2017 and is planned to finish in December 2018 once the birth data of all participants are obtained. Participant data will be deidentified and assigned a number code to ensure confidentiality for each woman and baby.

Electronic data will be stored and saved on a password-protected computer. Hard (paper) copies of the consent form, questionnaire and data sheets from the ultrasound examination will be stored in a locked filing cabinet in the principal researcher's office. This office is a secure room within the ultrasound department of the Townsville Hospital. Only the principal researcher 
and members of the research team will have access to the data.

\section{Author affiliations}

${ }^{1}$ Ultrasound Department, The Townsville Hospital, Townsville, Queensland, Australia

${ }^{2}$ College of Public Health, Medical and Veterinary Sciences, James Cook University,

Townsville, Queensland, Australia

${ }^{3}$ Medical Imaging \& Radiation Sciences, Monash University, Melbourne, Victoria,

Australia

${ }^{4}$ Department of Obstetrics and Gynaecology, The Townsville Hospital, Townsville, Queensland, Australia

${ }^{5}$ Department of Neonatology, The Townsville Hospital, Townsville, Queensland, Australia

${ }^{6}$ Mothers and Babies Research Centre, Hunter Medical Research Institute, John Hunter Hospital, The University of Newcastle, Newcastle, Australia

Contributors SB conceived the study and drafted the study design under the supervision of YK, DW, DR and MS. All authors contributed to the conception, design and development of the study protocol. MS provided her expertise for the study design, ethics and critical revision of the manuscript. DW provided his expertise for the ultrasound protocol, recruitment of participants and revision of the protocol. YK provided his expertise for ethics, statistics and drafting of the study protocol. DR provided her expertise for the study design, data management and analysis. All authors approved the final version and agree to be accountable for the contents and integrity of this manuscript.

Funding This study is partly funded by a Townsville Hospital and Health Service Research Grant.

Competing interests None declared.

Ethics approval This study was approved by the Townsville Health District Human Research Ethics Committee.

Provenance and peer review Not commissioned; externally peer reviewed.

Open Access This is an Open Access article distributed in accordance with the Creative Commons Attribution Non Commercial (CC BY-NC 4.0) license, which permits others to distribute, remix, adapt, build upon this work non-commercially, and license their derivative works on different terms, provided the original work is properly cited and the use is non-commercial. See: http://creativecommons.org/ licenses/by-nc/4.0/

(c) Article author(s) (or their employer(s) unless otherwise stated in the text of the article) 2017. All rights reserved. No commercial use is permitted unless otherwise expressly granted.
REFERENCES

1. Lim SS, Vos T, Flaxman AD. A comparative risk assessment of burden of disease and injury attributable to 67 risk factors and risk factor clusters in 21 regions, 1990-2010: a systematic analysis for the global burden of disease study 2010. Lancet 2012;380-:2224-60.

2. Hinchliffe SA, Lynch MR, Sargent PH, et al. The effect of intrauterine growth retardation on the development of renal nephrons. $\mathrm{Br} \mathrm{J}$ Obstet Gynaecol 1992;99:296-301.

3. Mañalich R, Reyes L, Herrera M, et al. Relationship between weight at birth and the number and size of renal glomeruli in humans: a histomorphometric study. Kidney Int 2000;58:770-3.

4. Hoy WE, Rees M, Kile E, et al. A new dimension to the Barker hypothesis: low birthweight and susceptibility to renal disease. Kidney Int 1999;56:1072-7.

5. Bagby SP. Developmental origins of renal disease: should nephron protection begin at birth? Clin J Am Soc Nephrol 2009;4:10-13.

6. Kandasamy $\mathrm{Y}$, Smith R, Wright IM. Oligonephropathy of prematurity. Am J Perinatol 2012;29:115-20.

7. Moritz KM, Caruana G, Wintour EM. Development of the kidneys and urinary tract. In: Rodeck $\mathrm{CH}$, Whittle MJ, eds. Fetal medicine: basic science and clinical practice. Sydney: Elsevier, 2009.

8. Saxén L, Sariola H. Early organogenesis of the kidney. Pediatr Nephrol 1987;1:385-92.

9. Hinchliffe SA, Sargent PH, Howard CV, et al. Human intrauterine renal growth expressed in absolute number of glomeruli assessed by the disector method and Cavalieri principle. Lab invest 1991;64:777-84.

10. De Curtis M, Rigo J. Nutrition and kidney in preterm infant. J Matern Fetal Neonatal Med 2012;25(sup1):55-59.

11. Zhang Y, Li H, Liu SJ, et al. The associations of high birth weight with blood pressure and hypertension in later life: a systematic review and meta-analysis. Hypertens Res 2013;36:725-35.

12. Luyckx VA, Bertram JF, Brenner BM, et al. Effect of fetal and child health on kidney development and long-term risk of hypertension and kidney disease. Lancet 2013;382:273-83.

13. Brennan S, Watson D, Rudd D, et al. Evaluation of fetal kidney growth using ultrasound: a systematic review. Eur J Radiol 2017;96:55-64.

14. Bakker J, Olree M, Kaatee R, et al. In vitro measurement of kidney size: comparison of ultrasonography and MRI. Ultrasound Med Biol 1998;24:683-8

15. Hadar E, Davidovits M, Mashiach R, et al. Sonographic evaluation of kidney parenchymal growth in the fetus. Arch Gynecol Obstet 2012;286:867-72.

16. Brennan S, Kandasamy Y. Renal parenchymal thickness as a measure of renal growth in low-birth-weight infants versus normalbirth-weight infants. Ultrasound Med Biol 2013;39:2315-20.

17. Eze CU, Akpan VP, Nwadike IU. Sonographic assessment of normal renal parenchymal and medullary pyramid thicknesses among children in Enugu, Southeast, Nigeria. Radiography 2016;22:25-31. 